\title{
Psychiatric Symptoms in Refractory Epilepsy During the First Year After Surgery
}

\author{
Sònia Ramos-Perdigués ${ }^{1,2}$ (D) Eva Baillés ${ }^{3,4} \cdot$ Anna Mané $^{5,6} \cdot$ Mar Carreño $^{7,8,9} \cdot$ Antonio Donaire $^{7,8,9} \cdot$ Jordi Rumià ${ }^{7,8}$. \\ Nuria Bargalló ${ }^{7,8,9} \cdot$ Teresa Boget ${ }^{7,8,9} \cdot$ Xavier Setoain ${ }^{7,8,9} \cdot$ Manuel Valdés $^{7} \cdot$ Luís Pintor $^{7,8,9}$
}

Published online: 31 July 2018

(C) The American Society for Experimental NeuroTherapeutics, Inc. 2018

\begin{abstract}
Psychiatric morbidity in drug-resistant epilepsy is frequent. Surgery is the best therapeutic alternative for treating seizures, but the current evidence concerning the effects of surgery on psychiatric disorders (PDs) is inconclusive. We aim to clarify surgery's role in long-term PDs. Using a prospective controlled study, we analyzed the psychopathologic outcomes of patients with drug-resistant epilepsy, comparing those who underwent surgery to those who did not due to not being suitable. Surgical candidates were paired ( $n=84)$ with the immediately following nonsurgical candidates $(n=68)$. Both groups continued their usual medical treatment. We studied psychiatric changes for each group and analyzed de novo and remission cases. The assessments were made during the presurgical evaluation, and at 6 months (6-M) and 12 months (12-M) after surgery. Finally, we determined associated factors for postsurgical PDs. At 12 months, using the Hospital Anxiety and Depression Scale (HADS), anxiety improved in both groups ( $p=$ $0.000)$, while depression improved only in the surgical group $(p=0.016)$. Moreover, all symptom dimensions on the Symptom Checklist-90-R (SCL-90), as well as severity, distress, and total symptoms, decreased only in the surgical group. These ameliorations reached not only statistical significance but also clinical significance for depression (HADS) $(p=0.014)$ and the interictal dysphoric disorder $(p=0.013)$. The main predictors for PDs after surgery were as follows: the presurgical and 6-month psychiatric symptoms, the absence of surgery, seizure outcomes, and some antiepileptic and psychiatric drugs. This study provides evidence that surgery for epilepsy could have a role in improving some symptoms of psychiatric disorders 12-M after the surgery.
\end{abstract}

Key Words Neuropsychiatry $\cdot$ Psychopathology $\cdot$ Epilepsy comorbidity $\cdot$ Consultation liaison psychiatry $\cdot$ Neurosurgery.

Sònia Ramos-Perdigués

sonia.ramos.12@gmail.com

1 Sant Joan de Déu Terres de Lleida Hospital, Avinguda de la Canadiense, 28, 25001 Lleida, Catalonia, Spain

2 Nostra Senyora de Meritxell Hospital, Escaldes-Engordany AD700, Andorra

3 Department of Experimental and Health Sciences, University Pompeu Fabra, Barcelona 08002, Spain

4 Autonomous University of Barcelona, Barcelona 08193, Spain

5 Institute of Neuropsychiatry and Addictions, Parc de Salut Mar and Foundation IMIM, Barcelona 08003, Spain

6 Center for Biomedical Research in Mental Health Network (CIBERSAM), Madrid 28029, Spain

7 Clinical Institute of Neurosciences, Hospital Clinic of Barcelona, Barcelona 08036, Spain

8 Epilepsy Unit, Hospital Clínic de Barcelona, Barcelona 08036, Spain

9 Hospital Clinic of Barcelona, Biomedical Research Institute August Pi i Sunyer (IDIBAPS), Barcelona 08036, Spain

\section{Introduction}

Epilepsy is a chronic condition affecting nearly 4 to 10 in 1000 people in developed countries [1]. Despite pharmacological advances, 20 to $40 \%$ of people with epilepsy are refractory to treatment [2]. Surgery for intractable epilepsy is increasingly being undertaken [3] as the best therapeutic option for achieving seizure-free [4] status.

More than half of refractory patients present with psychiatric disorders [5], which have a negative effect on quality of life (QOL) [4, 6] even independent of seizures [7]. Psychiatric disturbances are also common after surgery, either emerging de novo or as an exacerbation of pre-existing symptoms [3, 4]. Despite these facts, there are no established guidelines for pre- or postsurgical psychiatric assessment [3].

Previous research studies that have analyzed psychiatric outcomes in patients who have undergone surgery have used heterogeneous assessment methods that have not always been 
validated in patients with epilepsy [3]. Moreover, atypical psychiatric presentations are not considered in standard psychiatric diagnostic classification systems, such as the Diagnostic and Statistical Manual of Mental Disorders (DSM)-IV [5]. Furthermore, prior research has exhibited methodological limitations, for example, using crosssectional analyses, studying small or heterogeneous groups, or longitudinal studies with a retrospective design that do not include a control group or that have a limited follow-up period $[4,8,9]$. Results from studies with a control group showed that the controls subjects had a lower severity of psychiatric disturbances compared to surgical candidates [10]. Therefore, all of these factors could lead to meta-analytic bias [4].

Our previous work, a 6-month (6-M) prospective followup controlled study [11], showed high rates of psychopathology in both control and treatment groups. We observed an initial significant reduction after surgery for anxiety and depression, according to the Hospital Anxiety and Depression Scale (HADS), and also reductions in some domains of the Symptom Checklist-90-R (SCL-90-R) only in the surgical group. However, the first months after surgery are stressful [12], and it is during the first year following surgery that psychiatric disorders are most likely to appear [13]. During this period, different stages have been described (early postoperative phase, coping phase, and the start of the reorientation phase). After 12 months (12-M) following surgery, postoperative adaptation is in the past for those who are seizurefree and in good psychiatric condition, but those who suffer from psychiatric complications tend to continue having psychiatric symptoms [12]. Because of this, our initial findings cannot be extrapolated to long-term results [12].

Taking into consideration the psychological outcomes obtained 6-M after surgery, we wanted to determine if these results would persist over time. This study aims to clarify the effect of surgery on long-term psychiatric disorders through a controlled study.

\section{Methods}

\section{Design}

Through a prospective controlled study, we investigated patients with drug-resistant epilepsy, and we compared the psychopathological outcomes of those who underwent surgery to those who did not at three time points: baseline, 6-M after surgery, and 12-M after surgery.

\section{Setting and Participants}

This study was carried out at Barcelona's Hospital Clinic (2006-2012) and it was approved by the clinic's research ethics committee.
The epilepsy unit receives referrals of patients with epilepsy from all of Spain. In the neurology outpatient clinic, all patients with resistant epilepsy (that is, failure to achieve sustained seizure freedom following adequate trials of two antiepileptic drug schedules, whether as monotherapies or in combination) [14] who wished to be operated on were referred for a 1-week admission to the neurology ward. All gave informed consent and underwent a comprehensive presurgical assessment protocol. The presurgical protocol was completed during the admission procedure and included:

a) neuropsychological assessment,

b) psychiatric evaluation,

c) clinical history and neurological examination,

d) long-term video electroencephalogram,

e) structural magnetic resonance imaging (MRI) or functional MRI, and

f) fludeoxyglucose F18 positron emission tomography.

Accepting an alpha risk of 0.05 and a beta risk of less than 0.20 in a bilateral contrast, we needed 35 subjects in the first group and 35 in the second group to detect a difference greater than or equal to 2 units. It was assumed that the common standard deviation was $4.14[15,16]$. The estimated rate of loss at follow-up was $10 \%$.

Suitability for surgery was studied by the epilepsy committee, which comprised neurologists, neurosurgeons, neuropsychologists, neuroradiologists, psychiatrists, psychologists, and nuclear medicine specialists. Patients who presented with any of the following potentially confounding comorbidities were excluded: severe medical pathology, intellectual disability that would prevent patients answering all questionnaires on their own, severe dementia, nonepileptic seizures in addition to epilepsy, and previous surgery for epilepsy.

The flow chart in Fig. 1 shows the recruitment process.

\section{Clinical Assessments}

We collected sociodemographic, neurological, and psychiatric variables (see Table 1). We also assessed antiepileptic (every drug) and psychotropic treatments (clustered as antidepressants, antipsychotics, benzodiacepines, and pregabalin and gabapentin [PGB and GBP] when prescribed by a psychiatrist. Psychological interventions were not collected. Psychiatric evaluations were performed by one experienced psychiatrist, and they included DSM-IV Axis-I disorders and specific psychiatric conditions associated with epilepsy, such as interictal dysphoric disorder (IDD) [17] and interictal psychotic disorder (IPD) [3]. Self-assessment scales were completed by the patients under medical supervision. 
Patients included

Patients excluded

Patients who rejected to join in the study $(n=42) 7.9 \%$ Patients excluded after exclusion criteria $(n=326) 61.3 \%$

- Severe medical pathology $(n=68) 12.8 \%$

- Intellectual disability IQ > 70* $(n=157) 29.5 \%$

- Severe dementia ( $n=12) 2.3 \%$

- Epilepsy and non-epileptic seizures ( $n=65) 12.2 \%$

- Previous surgery for epilepsy $(n=24) 4.5 \%$

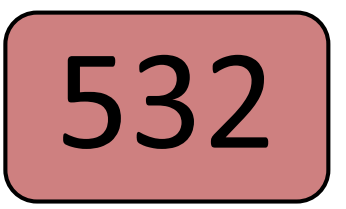

Patients admitted for pre-surgical assessment

(fulfilled inclusion criteria and underwent pre-surgical protocol)

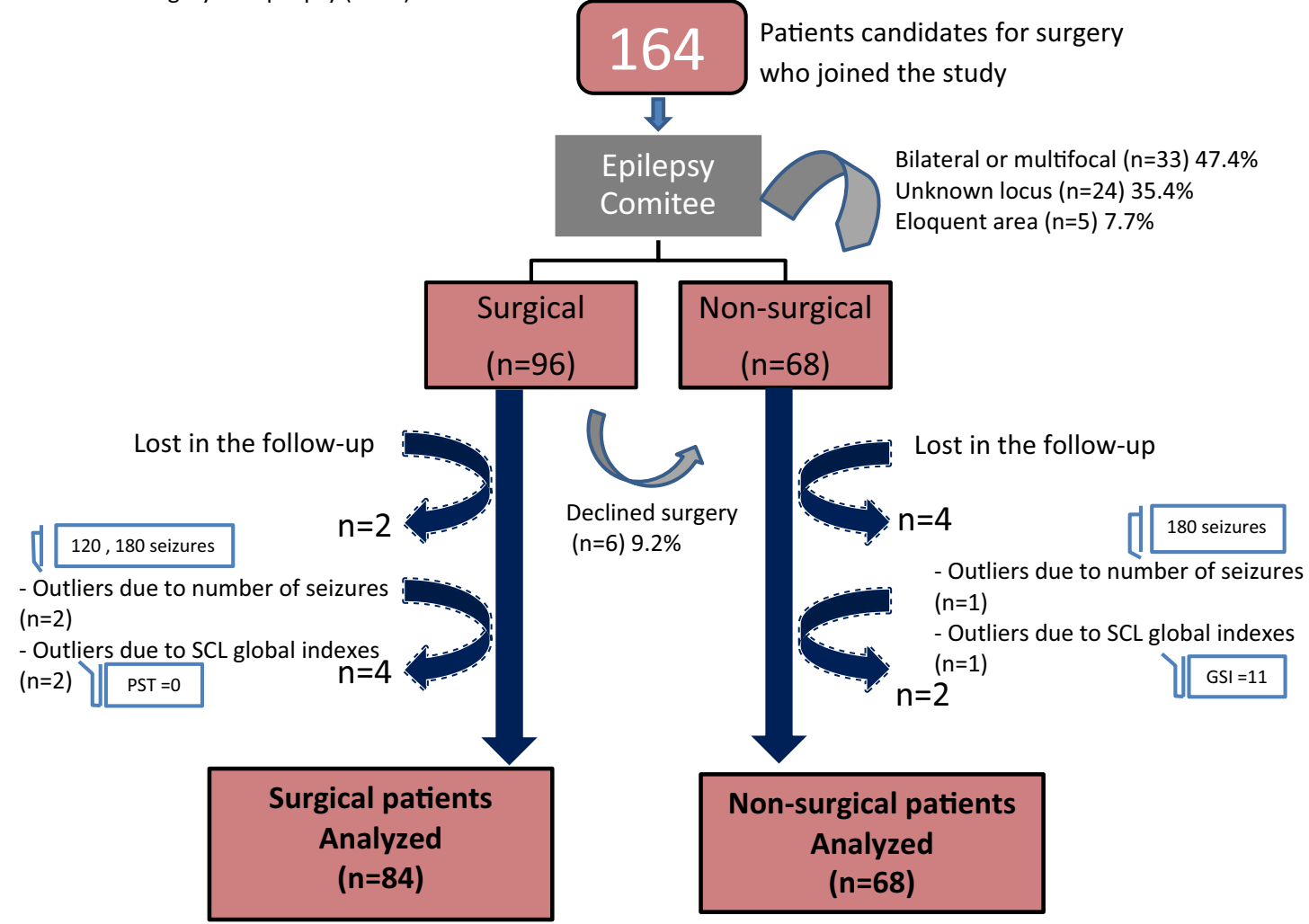

*Patients with mild intellectual disability who were able to answer all questionnaires on their own were not excluded

Fig. 1 Recruitment process

\section{Assessment Instruments}

The clinician version of the Structured Clinical Interview for DSM-IV (SCID-CV; Spanish version) [18] was used to assess DSM-IV Axis-I disorders. We grouped psychiatric disorders into six clusters: (1) affective disorders, including major depressive episodes, recurrent depression, dysthymic disorder, affective disorder due to a medical condition or substance disorder, adjustment disorder, and bipolar disorder; (2) anxiety disorders, including panic disorder, phobia, obsessive-compulsive disorder, post-traumatic stress disorder, and other anxiety disorders; (3) schizophrenia or schizoaffective disorder and other psychosis; (4) eating disorders; (5) conduct disorder; and (6) substance use disorder.
IDD was characterized by the presence of three out of eight symptoms: depressive mood, anergia, pain, insomnia, fear, anxiety, irritability, and euphoric mood [17].

IPD was defined as a state in which persistent or recurrent delusions, hallucinations, thought disorder, or severe behavioral abnormalities may be present in clear consciousness. It was considered interictal if the episode occurred during a seizure-free period or between habitual seizures with no distinct preceding seizures [3].

The Spanish version of HADS [19] was used as a selfassessed screening tool for anxiety and depression. It contained 14 items ( 7 pertaining to depression and 7 pertaining to anxiety). Each item was scored 0 to 3 . The cut-off point was $8 / 21$ for anxiety (HADS-A) and depression (HADS-D) [19]. 
Table 1 Sociodemographic, neurological, and psychiatric variables at baseline and differences between groups

\begin{tabular}{|c|c|c|c|c|c|c|c|c|c|c|c|c|}
\hline & \multicolumn{3}{|c|}{$\begin{array}{c}\text { TOTAL GROUP } \\
152\end{array}$} & \multicolumn{3}{|c|}{$\begin{array}{l}\text { SURGICAL GROUP } \\
84\end{array}$} & \multicolumn{3}{|c|}{$\begin{array}{c}\text { CONTROL GROUP } \\
68\end{array}$} & \multirow[t]{2}{*}{$\begin{array}{l}\text { (differences } \\
\text { groups) } \\
\mathrm{p}<0.005\end{array}$} \\
\hline & & & $\%$ & Mean & SD & $\%$ & Mean & SD & $\%$ & Mean & SD & \\
\hline \multicolumn{3}{|c|}{ SOCIODEMOGRAPHICS } & & & & & & & & & & \\
\hline \multirow{2}{*}{\multicolumn{3}{|c|}{ Age }} & & 37.17 & 11.00 & & 36.85 & 10.45 & & 37.49 & 11.66 & \\
\hline & & Gender (male) & 42.8 & & & 43.4 & & & 42.6 & & & \\
\hline \multirow{3}{*}{ Education } & Basic educatio & & 43.0 & & & 43.4 & & & 42.6 & & & \\
\hline & Secondary edu & cation & 41.7 & & & 42.9 & & & 39.7 & & & \\
\hline & Higher educati & & 15.2 & & & 13.3 & & & 17.6 & & & \\
\hline & Inactive & & 32.5 & & & 24.1 & & & 42.6 & & & \\
\hline Occupation & Housewife/stuc & dent & 17.2 & & & 19.3 & & & 14.7 & & & \\
\hline & Active & & 50.3 & & & 56.6 & & & 42.6 & & & \\
\hline Mrital state & Married & & 55.9 & & & 61.5 & & & 49.2 & & & \\
\hline Marital state & Separate/widoy & & 9.1 & & & 5.1 & & & 13.8 & & & \\
\hline & Single & & 35.0 & & & 33.3 & & & 36.9 & & & \\
\hline NEUROLOGICAL & & & & & & & & & & & & \\
\hline Idiopathic etiology & & & 68.3 & & & 66.8 & & & 71.7 & & & \\
\hline & More than one & type of seizures & 37.3 & & & 48.8 & & & 22.7 & & & $\mathrm{p}=0.001$ \\
\hline & & Tonico-clonic (TCG) & 21.9 & & & 17.9 & & & 26.5 & & & \\
\hline Types of epilepsy & Generalized & Others & 28.3 & & & 34.5 & & & 20.6 & & & \\
\hline & & Complex Partial (CPS) & 58.9 & & & 70.2 & & & 44.1 & & & $\mathrm{p}=0.003$ \\
\hline & Focal & Others & 24.3 & & & 23.8 & & & 25 & & & \\
\hline & Temporal & & 51.0 & & & 74.6 & & & 20.3 & & & \\
\hline & & Mesial & 34.7 & & & 54.2 & & & 9.4 & & & \\
\hline & & Neocortical & 5.4 & & & 9.6 & & & & & & \\
\hline & & Dual & 10.9 & & & 10.8 & & & 10.9 & & & \\
\hline & Multifocal & & 12.9 & & & 8.4 & & & 18.8 & & & \\
\hline Locus & Extratemporal & & 10.9 & & & 15.7 & & & 4.7 & & & \\
\hline & & Lobar & 2.2 & & & 2.5 & & & 1.5 & & & \\
\hline & & Sublobar & 6.6 & & & 10.9 & & & 1.6 & & & \\
\hline & & \begin{tabular}{|l} 
Multilobar \\
\end{tabular} & 2.1 & & & 2.4 & & & 1.6 & & & \\
\hline & Unknown or bi & ilateral & 25.2 & & & 1.2 .4 & & & 56.3 & & & $\mathrm{p}=0.000$ \\
\hline & Right & & 50.0 & & & 56.4 & & & 42.2 & & & \\
\hline Hemisphere & Left & & 34.5 & & & 35.9 & & & 32.8 & & & \\
\hline & Bilateral & & 11.3 & & & 7.7 & & & 15.6 & & & \\
\hline & Unknown & & 4.2 & & & 0 & & & 9.4 & & & $\mathrm{p}=0.013$ \\
\hline Age (month) of epi & epsy onset & & & 192.78 & 168.87 & & 173.59 & 138.81 & & $\begin{array}{c}215.2 \\
6\end{array}$ & 199.10 & \\
\hline $\mathrm{n}^{\circ}$ crisis at the onse & & & & 1.31 & 0.73 & & 1.31 & 0.73 & & 1.31 & 0.74 & \\
\hline Age (month) first a & tiepileptic. & & & 203.55 & 160.39 & & 189.73 & 136.23 & & $\begin{array}{c}224.6 \\
9\end{array}$ & 191.18 & \\
\hline $\mathrm{n}^{\circ}$ crisis per month & at basal time & & & 13.87 & 20.70 & & 15.95 & 21.06 & & 11.34 & 20.13 & \\
\hline $\mathrm{n}^{\circ}$ antiepileptics at & asal time & & & 2.18 & 0.77 & & 2.23 & 0.81 & & 2.13 & 0.73 & \\
\hline PSYCHIATRIC AS & SESSMENT & & & & & & & & & & & \\
\hline & M - Somatizatio & & & 1.11 & 0.72 & & 1.07 & 0.74 & & 1.15 & 0.69 & \\
\hline SCL-90-R & C-Obsessive-C & ompulsive & & 1.63 & 0.84 & & 1.68 & 0.85 & & 1.58 & 0.83 & \\
\hline & T-Interpersonal & Sensitivity & & 1.13 & 0.73 & & 1.10 & 0.75 & & 1.17 & 0.72 & \\
\hline & P - Depression & & & 1.34 & 0.76 & & 1.33 & 0.80 & & 1.35 & 0.70 & \\
\hline & $\mathrm{X}$ - Anxiety & & & 1.05 & 0.66 & & 1.03 & 0.67 & & 1.08 & 0.65 & \\
\hline & S - Hostility & & & 0.88 & 0.66 & & 0.82 & 0.63 & & 0.95 & 0.69 & \\
\hline & O - Phobic Anxi & & & 0.66 & 0.66 & & 0.72 & 0.65 & & 0.60 & 0.67 & \\
\hline & R - Paranoid Ide & ation & & 1.04 & 0.80 & & 0.95 & 0.77 & & 1.15 & 0.83 & \\
\hline & Y - Psychoticism & & & 0.72 & 0.55 & & 0.67 & 0.48 & & 0.77 & 0.62 & \\
\hline & I - Global severi & ity index & & 1.11 & 0.56 & & 1.08 & 0.55 & & 1.14 & 0.57 & \\
\hline & DI - Positive Syr & mptom Distress Index & & 1.97 & 0.48 & & 1.96 & 0.52 & & 1.98 & 0.45 & \\
\hline & T - Positive Sym & ptom Total & & 48.14 & 17.88 & & 47.24 & 17.46 & & 49.18 & 18.44 & \\
\hline HAD & D-D & & 36.6 & 4.80 & 4.14 & 32.4 & 5.24 & 4.40 & 43.2 & 4.27 & 3.76 & \\
\hline SCALE & $\mathrm{D}-\mathrm{A}$ & & 14.6 & 6.95 & 3.66 & 14.0 & 7.12 & 3.69 & 15.4 & 6.74 & 3.64 & \\
\hline SCID & ID affective diso & order & 21.6 & & & 23.5 & & & 19.4 & & & \\
\hline & ID psychotic dis & order & 4.1 & & & 4.9 & & & 3 & & & \\
\hline & ID anxiety disor & & 15.5 & & & 16.0 & & & 14.9 & & & \\
\hline & ID eating disord & & 0 & & & 0 & & & 0 & & & \\
\hline & ID drug use & & 1.4 & & & 0 & & & 3 & & & \\
\hline & ID conduct disol & rder & 0 & & & 0 & & & 0 & & & \\
\hline Psychiatric & Interictal Dysp & horic Disorder - IDD & 26.6 & & & 27.8 & & & 25 & & & \\
\hline $\begin{array}{l}\text { conditions associated } \\
\text { with epilepsy }\end{array}$ & Interictal Psych & hotic Disorder- IPD & 0 & & & 0 & & & 0 & & & \\
\hline & Antidepressant & & 16.4 & & & 17.9 & & & 14.7 & & & \\
\hline & Neuroleptics & & 2.6 & & & 1.2 & & & 4.4 & & & \\
\hline Psychiatric & Benzodiacepin & & 0.4 & & & 0 & & & 1.5 & & & \\
\hline & Pregabalin and & gabapentin & 15.7 & & & 16.4 & & & 15.0 & & & \\
\hline & No psychiatric & treatment & 68.8 & & & 67.2 & & & 70.7 & & & \\
\hline
\end{tabular}

The table is divided into three columns: The first describes the whole sample, the second the group who underwent surgery, and the third the nonsurgical group

Rows are clustered according to sociodemographic, neurological, and psychiatric variables

$\mathrm{SD}=$ standard deviation; No. $=$ number of subjects; $\%=$ percentage of subjects 
The SCL-90-R questionnaire included 90 items, each rated 0 to 4 [4]. Three indexes were calculated: the global severity index (GSI), which measured the global severity of psychiatric symptoms; the positive symptom total (PST), which considered the total number of symptoms, independent of their severity; and the positive symptom distress index (PSDI), which indicated the intensity of symptoms as perceived by patients' $[20,21]$.

All assessments were carried out during the initial presurgical evaluation and at 6-M and 12-M after surgery.

\section{Procedure}

All drug-resistant epileptic patients who decided to undergo surgery were admitted for a wider protocolized study, to which they gave informed consent. Based on the complete protocol outcomes, suitability for surgery was decided by the epilepsy committee at a weekly meeting. Each surgical candidate was paired with the immediately following nonsurgical candidate. Thereby, all patients were chronologically paired and all assessments were performed maintaining the same time interval with respect to the surgery.

Cases were reviewed at 6-M and 12-M after surgery, and controls were reviewed at the same 6-M and 12-M time points as their matched pairs. The postoperative assessment took place at an outpatient psychiatry clinic and involved psychiatric evaluation (SCID, HADS, SCL-90-R, IPD, and IDD) and seizure outcome (seizure frequency and Engel classification).

During the follow-up period, all patients maintained the same medication regimen as before surgery.

\section{Statistical Analysis}

First, variable distributions were examined, and appropriate tests were used accordingly. We carried out a descriptive analysis of all of the variables of interest. Subsequently, we compared base variables between the two groups to determine whether initial differences existed from the beginning of the study using a Student's $t$ test, chi-square test, analysis of variance (ANOVA), and Wilcoxon and Kruskal-Wallis tests. If any difference was detected, we examined how it influenced psychiatric complications.

Second, we compared cases and controls using paired $t$ tests and Wilcoxon's signed rank test to examine changes in psychiatric variables over time in each subject. Adjustments for multiple tests were performed using the Bonferroni procedure. In addition, we compared groups with regard to clinically relevant differences at $12-\mathrm{M}$. We considered de novo, remission, and no change for Axis-I diagnoses, IDD, and IPD, according to their definitions [17]. Also, significant changes in quantitative variables (SCL-90-R and HADS) were clustered, according to the literature, into three categories: no change, significant clinical improvement, and significant clinical deterioration [16].

To identify the effect of time and surgery interaction, we conducted a mixed-design ANOVA.

Finally, after examining the variables of interest using Pearson and Spearman correlations to explain psychiatric disturbances at $12-\mathrm{M}$, those that reached statistical significance were introduced into stepwise multiple regression analyses. Multiple regression was used to determine the contribution of each variable to psychopathologic results at 12-M.

Statistical analyses were carried out in Version 21 of SPSS for Windows.

\section{Results}

Of 164 eligible patients, 158 completed the psychiatric assessment at the follow-up visit at 12-M. There were no statistical differences in sociodemographic, clinical, or pathological variables between included patients and patients who did not complete the follow-up $(n=6)$. Moreover, 6 cases were excluded due to being outliers in some aspect (for example, the frequency of seizures or SCL-90-R ratings at 12-M), so the final sample consisted of 84 patients who underwent surgery and continued with their usual antiepileptic drugs (AEDs) (cases) and 68 patients who were not suitable for surgery but who also continued with their usual AEDs (controls).

\section{Descriptive Analysis at Baseline and Comparison Between Groups}

Sociodemographic, neurological, and psychiatric variables at baseline and the differences between groups are shown in (Table 1).

With regard to antiepileptic and psychiatric treatments we did not find any differences between groups.

As we found baseline differences in the type and location of seizures, we compared whether existing differences in psychiatric disorders depended on the presence versus absence of complex partial seizures (CPS), hemisphere (left $v s$ right), loci (temporal, multifocal, extratemporal, unestablished, or bilateral), or more than one type of seizure. The psychopathological differences evaluated were the global SCL and subscales, HADS, SCID diagnoses, IPD, and IDD. We did not find any psychopathological differences when we compared these groups.

There were no statistically significant differences with regard to prescribed drugs between surgical and nonsurgical patients with presurgical anxiety or depression ( $p=$ 0.163 ) and patients without presurgical anxiety or depression $(p=0.324)$. 


\section{Changes 12 Months After Surgery}

Regarding neurological variables, the number of seizures diminished significantly in the surgical group $(p=0.000)$, but not in the control group $(p=0.398)$. Groups differed in Engel classification $(p=0.000)$. We observed differences in the number of seizures per month (mean, 8.66; standard deviation [S.D.] $=15.50$ for controls $v s$ mean, 1.63; S.D. $=5.71$ for treatment cases; $p=0.006$ ). We compared whether psychopathological changes from the baseline measurement to the 12-M time point were dependent on the outcome of seizures. There were no statistically significant changes for any of the SCID diagnoses assessed, IDD or IPD among patients who were free from disabling seizures or among patients who suffered from disabling seizures. However, when we compared changes in quantitative scales, we determined that there were statistically significant changes in the HADS anxiety and depression scores for patients who were free from disabling seizures, and for all SCL-90 domains, with the exception of depression and hostility domains. We also found significant changes for anxiety (SCL-90 and HADS) in patients who were not free from disabling seizures. (See supplementary Table 1.)

We described the changes in psychopathology for each group (surgical and nonsurgical) independently. We tested each subject twice (repeated measures) at the baseline and at $12-\mathrm{M}$ after surgery, and we reported the differences. The significance of the differences is shown in Table 2.

Table 3 shows the comparison between the surgical and control groups with regard to relevant clinical changes (that is, de novo vs remission $v s$ no change) for SCID, IPD, and IDD. It also shows the clustering of the changes for SCL and HADS subscales into three categories (that is, no change, relevant clinical improvement, and relevant clinical deterioration) considering the minimal differences perceived by patients and labeled as the minimal important difference (MID) according to literature [16].

There were no statistically significant differences at $12-\mathrm{M}$ with regard to prescribed drugs between surgical and nonsurgical patients with presurgical anxiety or depression $(p=$ 0.606 ) and patients without presurgical anxiety nor depression $(p=0.371)$.

\section{Effect of Surgery and Time Interaction on Psychiatric Outcomes}

Table 2 shows significant changes over time in the psychiatric variables that differed between groups. To determine the effect of the interaction between the two variables (time and surgery), we conducted a mixed-design ANOVA. Only HADS$\mathrm{D}$, paranoidism, GSI, and PST showed significant changes due to the interaction effect. The complete results are shown in Table 4.

\section{Prediction at 12 Months After Surgery}

We initially ran univariate analyses for all the independent variables that could biologically explain the changes in psychiatric symptoms: age, sex, type of epilepsy, location, laterality, etiology, age at epilepsy onset, pre-assessment seizure outcomes, history of prior psychiatric disorders, prior psychiatric outcomes, epilepsy treatment, psychiatric treatment, and the surgical condition. The variables that reached significance in the univariate analyses were included in the multiple regression analyses.

Stepwise multiple regressions were performed initially for GSI, PST, and PSDI at the 12-M interval. For GSI at 12-M, we introduced the following: age, age at epilepsy onset, number of seizures at $12-\mathrm{M}$, carbamazepine and topiramate use, antidepressant treatment, baseline and 6-M GSI, prior psychiatric disorders, and surgical condition. Only GSI at 6-M $(\beta=0.509$, $95 \%$ confidence intervals $[\mathrm{CI}][0.309,0.671], p=0.000)$, baseline GSI $(\beta=0.351,95 \%$ CI $[0.192,0.622], p=0.000)$, and surgical condition $(\beta=-0.147,95 \%$ CI $[-0.314$, 0.008], $p=0.040$ ) were included in the final model.

For PST at 12-M, after including age, age at epilepsy onset, carbamazepine and topiramate use, no psychiatric treatment, PGB and GBP, baseline and 6-M PST, prior psychiatric disorders, and surgical condition in the model, only PST at 6-M $(\beta=0.586,95 \%$ CI $[0.417,0.783], p=0.000)$, baseline PST $(\beta=0.273,95 \%$ CI $[0.121,0.545], p=0.003)$, and carbamazepine $(\beta=-0.146,95 \%$ CI $[-11.655,-0.430], p=0.035)$ contributed to predicting the final model.

In the case of PSDI at 12-M, the following variables were included: age at epilepsy onset, number of seizures at 12-M, surgical condition, prior psychiatric disorders, and baseline and 6-M PSDI. Only the baseline PSDI $(\beta=0.471,95 \% \mathrm{CI}$ $[0.275,0.780], p=0.000)$ and $6-\mathrm{M}$ PSDI $(\beta=0.333,95 \% \mathrm{CI}$ $[0.108,0.560], p=0.004)$ remained in the final model.

With regard to the HADS, stepwise multiple regressions were also performed. For HADS-D, we introduced into the model the number of seizures at 6-M and 12-M; Engel classification; valproate, carbamazepine, and levetiracetam use; PGB and GBP, HADS-D, and HADS-A at baseline and at 6M; HADS-A at 12-M; prior psychiatric disorders; and surgical condition. Only the 6-M HADS-D $(\beta=0.487,95 \%$ CI $[0.290$, $0.594], p=0.000)$, levetiracetam $(\beta=-0.214,95 \%$ CI $[-$ $2.594,-0.348], p=0.011)$, carbamazepine $(\beta=-0.204$, $95 \%$ CI $[-2.576,-0.263], p=0.017)$, PGB and GBP $(\beta=$ $0.193,95 \%$ CI $[0.027,0.220])$, prior psychiatric disorders $(\beta=0.182,95 \%$ CI $[0.094,2.399], p=0.034)$, and number of seizures at $6-\mathrm{M}(\beta=0.164,95 \% \mathrm{CI}[0.001,0.079], p=$ $0.046)$ were included in the final model. In the case of the HADS-A subscale, we introduced age, number of seizures at 6-M and 12-M, prior psychiatric disorders, no psychiatric treatment, surgical condition, baseline and 6-M HADS-A, and baseline, 6-M, and 12-M HADS-D. Only the 6-M 
Table 2 Quantitative psychopathological changes at 12 months following surgery

\begin{tabular}{|c|c|c|c|c|c|c|c|c|c|c|}
\hline & \multicolumn{5}{|c|}{ CONTROL } & \multicolumn{5}{|c|}{ SURGICAL } \\
\hline & \multicolumn{2}{|c|}{ Results at $12-\mathrm{M}$} & \multicolumn{3}{|c|}{ Differences (basal-12month) } & \multicolumn{2}{|c|}{ Results at $12-\mathrm{M}$} & \multicolumn{3}{|c|}{ Differences (basal-12month) } \\
\hline & MEAN & SD & MEAN & SD & $\mathrm{p}$ & MEAN & $\mathrm{SD}$ & MEAN & SD & $\mathrm{p}$ \\
\hline \multicolumn{11}{|l|}{ SCL-90- R SCALE } \\
\hline SOM - Somatization & 0.94 & 0.73 & 0.16 & 0.58 & 0.069 & 0.69 & 0.63 & 0.44 & 0.68 & $0.000 * *$ \\
\hline O-C - Obsessive-Compulsive & 1.43 & 0.87 & 0.07 & 0.60 & 0.262 & 1.27 & 0.96 & 0.42 & 0.90 & $0.001 * *$ \\
\hline INT -Interpersonal Sensitivity & 0.93 & 0.74 & 0.14 & 0.57 & 0.111 & 0.84 & 0.69 & 0.33 & 0.58 & $0.000 * *$ \\
\hline DEP - Depression & 1.12 & 0.85 & 0.13 & 0.64 & 0.097 & 0.99 & 0.97 & 0.34 & 0.70 & $0.001 * *$ \\
\hline ANX - Anxiety & 0.83 & 0.56 & 0.14 & 0.50 & 0.011 & 0.71 & 0.73 & 0.35 & 0.64 & $0.000 * *$ \\
\hline HOS - Hostility & 0.63 & 0.50 & 0.17 & 0.65 & 0.117 & 0.48 & 0.52 & 0.33 & 0.63 & $0.000 * *$ \\
\hline PHO - Phobic Anxiety & 0.50 & 0.66 & 0.01 & 0.59 & 0.891 & 0.40 & 0.67 & 0.30 & 0.65 & $0.000 * *$ \\
\hline PAR - Paranoid Ideation & 0.95 & 0.80 & 0.08 & 0.73 & 0.264 & 0.63 & 0.67 & 0.35 & 0.65 & $0.000 * *$ \\
\hline PSY - Psychoticism & 0.48 & 0.44 & 0.16 & 0.40 & 0.008 & 0.43 & 0.52 & 0.23 & 0.44 & $0.000 * *$ \\
\hline GSI- Global Severity Index & 0.91 & 0.56 & 0.13 & 0.38 & 0.014 & 0.75 & 0.61 & 0.35 & 0.47 & $0.000 * *$ \\
\hline $\begin{array}{l}\text { PSDI - Positive Symptom } \\
\text { Distress Index }\end{array}$ & 1.85 & 0.50 & 0.05 & 0.41 & 0.422 & 34.49 & 21.06 & 13.22 & 16.99 & $0.000 * *$ \\
\hline PST - Positive Symptom Total & 42.39 & 19.66 & 4.66 & 13.12 & 0.023 & 0.69 & 0.63 & 0.44 & 0.68 & $0.000 * *$ \\
\hline \multicolumn{11}{|l|}{ HAD SCALE } \\
\hline HAD-D & 4.53 & 3.64 & -1.13 & 3.95 & 0.063 & 3.42 & 3.69 & 1.65 & 4.39 & $0.016^{*}$ \\
\hline HAD-A & 0.33 & 0.48 & 5.82 & 2.99 & $0.000 *$ & 0.34 & 0.48 & 6.69 & 3.59 & $0.000 *$ \\
\hline
\end{tabular}

The table is divided into two columns. The first describes the group who underwent surgery and the second the nonsurgical group. Each subject was tested twice. The first columns of each group show the mean scores at 12 months, and the third columns of each group show the mean differences between the baseline and 12 month after surgery. The last columns of each group are $p$ values, and they indicate significant changes for within-group comparisons and were set considering Bonferroni correction: HADS $(p \leq 0.025 *)$, SCL-90 $(p \leq 0.004 * *)$

$\mathrm{SD}=$ standard deviation

HADS-D $(\beta=0.443,95 \%$ CI $[0.031,0.084], p=0.000)$ and no psychiatric treatment $(\beta=-0.215,95 \%$ CI $[-0471,-$ $0.014], p=0.038$ ) remained in the final model.

\section{Discussion}

The main finding in our study was that surgery for epilepsy could have a role in improving some symptoms of psychopathological disturbances at the 12-M time point. During the reorientation phase, psychiatric benefits and disturbances tend to persist. Therefore, our results at 12-M may correspond to stable conditions. At $12-\mathrm{M}$, we identified a significant reduction in all nine primary dimensions of SCL-90, as well as total symptoms, distress, and severity of symptoms. Moreover, anxiety and depression were also ameliorated (based on the HADS score). These improvements were only observed for the patients in the surgical group, except for anxiety levels, which also decreased for patients in the control group. Nevertheless, the control group did not experience amelioration for any other dimension, and IDD even showed an increase. These findings strengthened our 6-M results in the same sample [11]. The improvement was due to the fact that while some SCL-90 domains decreased initially and remained at that level, others, such as the obsessive-compulsive, interpersonal sensitivity, depression, hostility, and psychoticism, did not ameliorate until 12-M.

In addition, we compared changes at 12-M from a clinical perspective because statistical differences are not always perceived by patients. Therefore, we grouped together de novo versus remission cases for categorical variables and clinical improvement versus clinical deterioration for quantitative variables, considering minimal differences perceived by the patients, according to literature parameters [16]. We did not find any clinically significant improvements for any SCID diagnoses, SCL-90 subscales, or IPD. Nonetheless, it was only in the surgical group that the percentage of patients that improved exceeded those who worsened for IDD $(p=0.013)$ and HADS-D $(p=0.014)$. Despite finding fewer significant changes when considering clinical relevance, this is the first study to differentiate between statistical and clinical relevance. With this in mind, although we did not find changes in SCID 
Table 3 Qualitative psychopathological changes 12 months following surgery

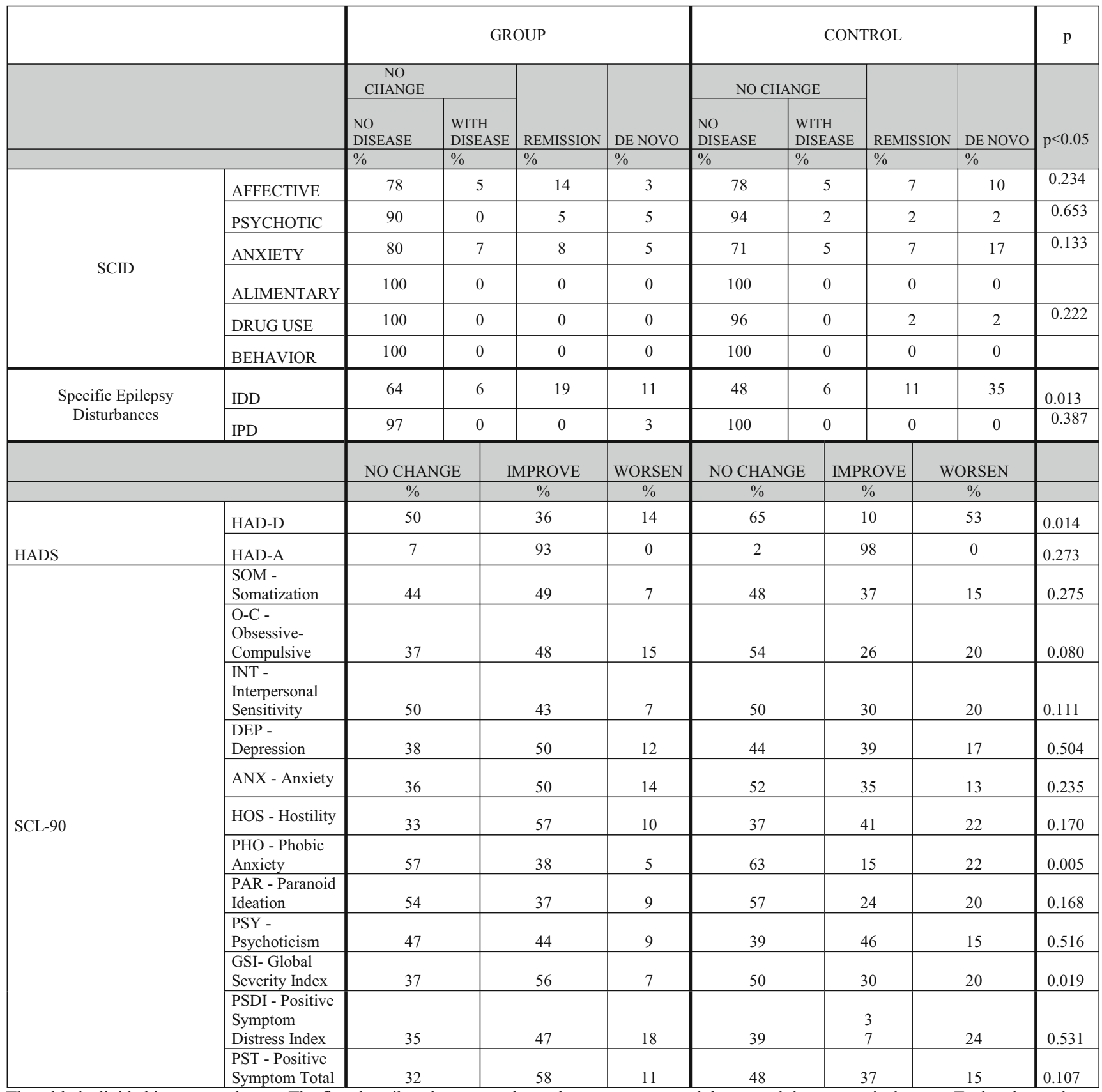

The table is divided into two columns. The first describes the group who underwent surgery and the second the nonsurgical group. Each column shows the patients who experienced no change and the patients who showed a significant clinical change from baseline. For categorical variables, no change was divided by no disease and with disease, while for quantitative variables the classification was done following numerical values. The $p$-values in the last column indicate the significance of the between-group comparisons and was set using Bonferroni corrections: SCID ( $p \leq 0.008)$, HADS, IDD and IPD $(p \leq 0.025)$, SCL-90 $(p \leq 0.004)$

$\%=$ percentage of subjects

diagnoses, patients may perceive an improvement in some psychiatric domains after surgery.

Psychiatric results following surgery have been controversial. While some authors have reported deterioration [22, 23] or no change $[22,24]$, others have found improvements in quantitative scales $[7,10,24-26]$ and categorical variables
$[25,27]$ (DSM diagnosis). Discrepancies could be due to methodological limitations, such as type II errors, noncorrected multiple comparisons, and the lack of a control group [22, 24, 28]. Having a control group helps to avoid misleading conclusions due to the variability in the individuals' changes over time and due to the anxiety-ridden state in 
Table 4 Effect of time-surgery interaction on the change in psychological outcomes. Mixed-design ANOVA

Mixed-design ANOVA

\begin{tabular}{llllll}
\hline Variable & \multicolumn{5}{l}{ Interaction effect of Surgery-Time } \\
\hline HAD Scale & df & MS & $F$ & $p$ value & $\eta_{\mathrm{p}}{ }^{2}$ \\
HAD-D & 1.844 & 61.805 & 7.158 & $0.001^{*}$ & 0.077 \\
HAD-A & 2 & 17.369 & 2.604 & 0.076 & 0.025 \\
SCL-90-R & df & MS & $F$ & $p$ value & $\eta_{\mathrm{p}}{ }^{2}$ \\
SOM & 2 & 0.264 & 1.958 & 0.144 & 0.022 \\
OC & 1.866 & 0.638 & 2.695 & 0.074 & 0.030 \\
INT & 2 & 0.246 & 1.555 & 0.214 & 0.018 \\
DEP & 2 & 0.252 & 1.215 & 0.299 & 0.014 \\
ANX & 2 & 0.346 & 2.418 & 0.092 & 0.027 \\
HOS & 2 & 0.253 & 1.470 & 0.233 & 0.017 \\
PHO & 1.848 & 0.569 & 3.048 & 0.054 & 0.034 \\
PAR & 2 & 0.797 & 3.995 & $0.020^{*}$ & 0.044 \\
PSY & 2 & 0.096 & 1.137 & 0.323 & 0.013 \\
GSI & 2 & 0.272 & 3.332 & $0.038^{*}$ & 0.037 \\
PSD & 2 & 0.150 & 1.869 & 0.160 & 0.021 \\
PST & 2 & 429.964 & 4.311 & $0.015^{*}$ & 0.048 \\
\hline
\end{tabular}

The table is divided into six columns. The first contains the psychiatric variables assessed. The other columns contain data on the surgery-time interaction analyzed with the mixed-design ANOVA. The $p$ values in the column indicate the significance of the surgery-time interaction, and values were set using Bonferroni's correction, which were automatically calculated by SPSS

$\mathrm{df}=$ degrees of freedom, MS = mean squared, $\eta_{\mathrm{p}}{ }^{2}=$ partial eta squared

* Statistically significant

the preoperative phase. This last aspect could explain the improvement in anxiety seen in the control group. We intended to overcome the limitations in the literature by using a prospective controlled design, using an appropriate sample size, determining similar baseline scores for both groups, and making corrections for multiple comparisons.

According to the literature, studies using SCL-90 reported improvements in symptomatology. Cunha's group [10] reported an improvement in all subscales except hostility and paranoid ideation at 6 months. We overcome some of their limitations by also assessing the control group twice, conducting follow-up over a longer period, and making corrections for multiple comparisons, with similar initial psychopathological scores in both groups. Other noncontrolled studies have conducted longer follow-ups, but have only reported GSI values [29]. In line with our results, Glosser et al. observed that although the prevalence of psychiatric complications (SCID) did not change, there was a significant decline in the severity of symptoms (quantitative variables). Despite this, Altshuler et al. [30] found postsurgical resolution of depressive episodes (SCID diagnosis) in almost $50 \%$ of patients. These rates are higher than ours because they considered lifetime depression rather than preoperative depression.

Regarding the time effect, some noncontrolled studies reproduced similar results to ours. Devinsky et al. [25] pointed out that the improvement in depression and anxiety symptoms occurred soon after surgery, with a maximal reduction at 3 months, and small increases at 12 months and 24 months. Also, Spencer reported an initial improvement in anxiety and depression, which was then lost for depression at 12 months [31]. Another controlled study, in line with ours, reported an improvement in depression, but not in anxiety [23].

Temporal lobe (TL) epilepsy is the most common and most surgically remediable form of epilepsy [32], and CPS mainly arises from the TL (60\%). Thus, it is logical to consider that TL epilepsy and CPS predominate in the surgical group. Some authors have not observed an association between psychiatric disturbances and location [3, 25], but others have [8]. The TL compromises the limbic system [30], in which dysfunction causes mood disturbances [13]. IDD has historically been related to TL [17], and it has achieved extraordinarily positive postsurgical outcomes not seen for other psychiatric disorders [33]. Although we did not observe a change in IDD at 6-M, we did obtain IDD amelioration in the surgical group and an IDD increase in the nonsurgical group at 12-M.

According to other studies [3,33], the existence of psychiatric disturbances is the strongest predictor of psychopathological problems at 12-M after surgery, as is also the case at 6-M after surgery. Thus, psychiatric history could be a risk factor for failing to cope with the reorientation phase, and 6-M outcomes predict later results. Notwithstanding, at 12-M, our mixed design ANOVA reflected a weak but significant effect of the interaction between surgery and time in terms of GSI, PST, depression, and paranoidism; surgery only attained significance as a protective factor for GSI. Considering all of the results, it is reasonable to suggest that surgery could have a role in ameliorating some psychiatric symptoms because only surgical patients obtained benefits. Despite this observation, prior psychiatric disorders and those measured at the 6-M interval acquired greater relevance in the multiple regression analyses. Other variables were found to have a protective role against depression, such as the administration of carbamazepine, which is consistent with its stabilizing properties [34], and with the administration of levetiracetam, despite the adverse behavioral effects reported with its use [35, 36]. Also, the absence of psychiatric treatment was a protective factor for anxiety and taking pregabalin and gabapentin [35] could worsen depression. This finding was probably due to the fact that the use of treatment correlated with weak premorbid mental health rather than the depressive effect of prebagalin or gabapentin.

Regarding seizure outcomes, we only noted seizure reduction and psychiatric disturbance amelioration in the surgical group. In the literature, results were controversial; while some studies $[8,37,38]$ found a correlation between 
psychopathology and seizure outcome, others did not $[25,26$, 28]. In our regression models, seizure outcome only reached significance in the prediction of HADS-D. These discrepancies could be due to the distinct seizure assessment.

Alternatively, surgery may play its own role by removing pathological tissue and improving the regulation of emotions [7]. Despite TL epilepsy having been historically associated with psychiatric disturbances, frontal lobe dysfunction has also been related with depression. There is limited research about the neurobiological mechanisms that cause psychiatric disorders. There is an inhibitory activity of serotonin and noradrenaline due to the excessive excitatory activity of the chronic seizure disorder. The decline of those neurotransmitters has been shown to facilitate the kindling process of seizure foci. To date, forced normalization is the most commonly attributed mechanism of mood disturbances. Surgery eradicates the mood stabilizing effect of seizures by seizure freedom $[6,17]$. This finding accords with the particular linkage of the psychiatric disorders when there is full control of seizures at 6-M, and it may explain why we found improvement at $12-\mathrm{M}$, when the homeostasis of the tissue activity returns after the forced normalization phase.

\section{Limitations}

Surgery depended on patient suitability and was not randomized due to ethical reasons. Although we took account of differences in laterality, loci, and type of seizures, the similar baseline scores for psychopathologies in both groups indicated equal severity. Also, regarding the design, there was no blinding process for controls or patients.

We used some assessment tools that have not been validated in epileptic patients. However, the interviews were performed by personnel trained in neurological diseases, and included specific epilepsy-related conditions. Moreover, SCL90-R has been proposed as a responsive tool for the assessment of epileptic patients [39].

Furthermore, although we explained to the patients in the first visit that they had to log the frequency of seizures each day before bedtime, we did not distribute a predesigned questionnaire for collecting seizure frequency, which could have led to recall and reporting bias.

Our hospital is a reference center in Spain for refractory epilepsy, and our role was to assess psychiatric symptoms in order to guarantee that symptoms were not causing a severe deterioration in functioning. To rule out risk of self-harm or harm to others, we advised patients to seek help from the reference team if any alarming symptoms emerged during the follow-up period. Indeed, we were aware that three visits were insufficient for an adequate follow-up, and that assistance should be provided by the patient's reference team. Our team provided additional support for the patients and for the regional teams. It has been reported that some psychiatric treatments could influence psychiatric outcomes $[9,31]$ and seizures [9]. Despite this, there were no differences between groups with regard to the psychiatric treatment; we clustered treatments into main groups, and we were unable conduct a more detailed study of the intrinsic properties of each drug. We neither reported the dosage nor considered the dose adjustment to be a major change, unlike a drug addition or cessation. Psychotherapies were not assessed due to the impossibility of controlling different interventions and associated variables when executed by the local teams. However, psychological interventions could have contributed to symptomatic improvement. Furthermore, desadaptative personality traits were not collected, although they could have influenced the lack of psychiatric improvement. Finally, despite the fact that the literature considers the postoperative adaptation phase to end after 12 months and because psychological functioning tends to persist over time, a longer follow-up would provide further insights into the long-term psychiatric sequelae.

\section{Conclusion}

This study shows that surgery for refractory epilepsy could have a role in ameliorating some psychopathological domains at $12-\mathrm{M}$ because psychiatric improvement was only observed in the surgical group. The strongest predictors for psychiatric disorders after surgery were previous psychiatric conditions and 6-M postsurgery psychiatric outcomes, seizure frequency, and antiepileptic and psychiatric treatment; the occurrence of surgery reached statistical significance as a weak protective factor for global severity of SCL-90. Additional research is warranted to broaden the knowledge of the impact of surgery in psychopathological domains.

Acknowledgments This study was supported by the Fondo de Investigación Sanitaria PI040418 (Spain).

\section{Compliance with Ethical Standards}

Required Author Forms Disclosure forms provided by the authors are available with the online version of this article.

Disclosures None of the authors has any conflict of interest to disclose.

\section{References}

1. Picot M-C, Baldy-Moulinier M, Daurès J-P, et al. The prevalence of epilepsy and pharmacoresistant epilepsy in adults: a populationbased study in a Western European country. Epilepsia 2008;49: 1230-1238.

2. French JA. Refractory epilepsy: clinical overview. Epilepsia 2007;48 Suppl 1:3-7. 
3. Foong J, Flugel D. Psychiatric outcome of surgery for temporal lobe epilepsy and presurgical considerations. Epilepsy Res. 2007;75:84-96.

4. Liu S-Y, Yang X-L, Chen B, et al. Clinical outcomes and quality of life following surgical treatment for refractory epilepsy: a systematic review and meta-analysis. Medicine (Baltimore). 2015;94: e500.

5. Hellwig S, Mamalis P, Feige B, et al. Psychiatric comorbidity in patients with pharmacoresistant focal epilepsy and psychiatric outcome after epilepsy surgery. Epilepsy Behav. 2012;23:272-279.

6. Kanner AM. Psychiatric issues in epilepsy: the complex relation of mood, anxiety disorders, and epilepsy. Epilepsy Behav. 2009;15: 83-87.

7. Meldolesi GN, Di Gennaro G, Quarato PP, et al. Changes in depression, anxiety, anger, and personality after resective surgery for drug-resistant temporal lobe epilepsy: a 2-year follow-up study. Epilepsy Res. 2007;77:22-30.

8. Wrench JM, Rayner G, Wilson SJ. Profiling the evolution of depression after epilepsy surgery. Epilepsia 2011;52:900-908.

9. Kanner AM, Palac S. Neuropsychiatric complications of epilepsy. Curr. Neurol. Neurosci. Rep. 2002;2:365-372.

10. Cunha I, Brissos S, Dinis M, et al. Comparison between the results of the Symptom Checklist-90 in two different populations with temporal lobe epilepsy. Epilepsy Behav. 2003;4:733-739.

11. Ramos-Perdigués S, Baillés E, Mané A, et al. A prospective study contrasting the psychiatric outcome in drug-resistant epilepsy between patients who underwent surgery and a control group. Epilepsia 2016;57:1680-1690.

12. Koch-Stoecker S, Schmitz B, Kanner AM. Treatment of postsurgical psychiatric complications. Epilepsia 2013;54:46-52.

13. Cleary RA, Baxendale SA, Thompson PJ, et al. Predicting and preventing psychopathology following temporal lobe epilepsy surgery. Epilepsy Behav. 2013;26:322-334.

14. Kwan P, Arzimanoglou A, Berg AT, et al. Definition of drug resistant epilepsy: consensus proposal by the ad hoc Task Force of the ILAE Commission on Therapeutic Strategies. Epilepsia [Internet]. 2010 [cited 2015 Dec 26];51:1069-1077. Available from: http:// www.ncbi.nlm.nih.gov/pubmed/19889013.

15. Breeman S, Cotton S, Fielding S, et al. Normative data for the Hospital Anxiety and Depression Scale. Qual. Life Res. 2015;24: 391-398.

16. Norman GR, Sloan JA, Wyrwich KW. Interpretation of Changes in Health-related Quality of Life. Med. Care 2003;41:582-592.

17. Blumer D, Montouris G, Davies K. The interictal dysphoric disorder: recognition, pathogenesis, and treatment of the major psychiatric disorder of epilepsy. Epilepsy Behav. 2004;5:826-840.

18. First M, Spitzer R, Gibbon Miriam, and Williams, Janet BW (1996) Structured Clinical Interview for DSM-IV Axis I Disorders, Clinician Version (SCID-CV). Washington, DC Am. ... [Internet]. [cited 2016 Feb 25]; Available from: https://scholar.google.es/ scholar?cluster $=6807271620914188016 \&$ hl $=$ es\&as_sdt $=$ $2005 \&$ sciodt $=0,5 \# 0$

19. Herrero MJ, Blanch J, Peri JM, et al. A validation study of the hospital anxiety and depression scale (HADS) in a Spanish population. Gen. Hosp. Psychiatry 2003;25:277-283.

20. Derogatis L. SCL-90-R: Administration, Scoring of Procedures Manual-II for the R (evised) Version and Other Instruments of the Psychopathology Rating Scale Series. 1992 [cited 2016 Feb 7]; Available from: https://scholar.google.es/scholar?hl=es\&q=scl$90+$ scale \&btnG $=\& 1 \mathrm{r}=\# 0$.

21. de Rivera JG, Derogatis L. The spanish version of the SCL-90-R. Normative data in the general population. Towson Clin. ...
[Internet]. 1989 [cited 2016 Feb 7]; Available from: https:// scholar.google.es/scholar?q=The+spanish+version+of+the+SCL$90-$ R. +Normative+data + in + the $\&$ btn $\mathrm{G}=\& \mathrm{hl}=$ es \&as_sdt $=0 \%$ 252C5\#0.

22. Bujarski KA, Hirashima F, Roberts DW, et al. Long-term seizure, cognitive, and psychiatric outcome following trans-middle temporal gyrus amygdalohippocampectomy and standard temporal lobectomy. J. Neurosurg. 2013;119:16-23.

23. Reuber M, Andersen B, Elger CE, et al. Depression and anxiety before and after temporal lobe epilepsy surgery. Seizure J. Br. Epilepsy Assoc. 2004;13:129-135.

24. Glosser G, Zwil AS, Glosser DS, et al. Psychiatric aspects of temporal lobe epilepsy before and after anterior temporal lobectomy. J. Neurol. Neurosurg. Psychiatry 2000;68:53-58.

25. Devinsky O, Barr WB, Vickrey BG, et al. Changes in depression and anxiety after resective surgery for epilepsy. Neurology 2005;65: 1744-1749.

26. Engman E, Malmgren K. A longitudinal study of psychological features in patients before and two years after epilepsy surgery. Epilepsy Behav. 2012;24:221-226.

27. Pintor L, Bailles E, Fernández-Egea E, et al. Psychiatric disorders in temporal lobe epilepsy patients over the first year after surgical treatment. Seizure 2007;16:218-225.

28. Ring HA, Moriarty J, Trimble MR. A prospective study of the early postsurgical psychiatric associations of epilepsy surgery. J. Neurol. Neurosurg. Psychiatry 1998;64:601-604.

29. Guangming Z, Wenjing Z, Guoqiang C, et al. Psychiatric symptom changes after corticoamygdalohippocampectomy in patients with medial temporal lobe epilepsy through Symptom Checklist 90 Revised. Surg. Neurol. 2009;72:587-591; discussion 591.

30. Altshuler L, Rausch R, Delrahim S, et al. Temporal lobe epilepsy, temporal lobectomy, and major depression. J. Neuropsychiatry Clin. Neurosci. 1999;11:436-443.

31. Spencer SS, Berg AT, Vickrey BG, et al. Initial outcomes in the Multicenter Study of Epilepsy Surgery. Neurology $2003 ; 61: 1680$ 1685.

32. Engel J. Surgery for seizures. N. Engl. J. Med. 1996;334:647-652.

33. de Filho GMA, Mazetto L, Gomes FL, et al. Pre-surgical predictors for psychiatric disorders following epilepsy surgery in patients with refractory temporal lobe epilepsy and mesial temporal sclerosis. Epilepsy Res. 2012;102:86-93.

34. Zhang Z-J, Tan Q-R, Tong Y, et al. The effectiveness of carbamazepine in unipolar depression: a double-blind, randomized, placebocontrolled study. J. Affect. Disord. $2008 ; 109: 91-97$.

35. Briggs DE, French JA. Levetiracetam safety profiles and tolerability in epilepsy patients. Expert Opin. Drug Saf. [Internet]. 2004;3: 415-424. Available from: http://www.ncbi.nlm.nih.gov/pubmed/ 15335297.

36. Andersohn F, Schade R, Willich SN, et al. Use of antiepileptic drugs in epilepsy and the risk of self-harm or suicidal behavior. Neurology 2010;75:335-340.

37. de Araújo Filho GM, Gomes FL, Mazetto L, et al. Major depressive disorder as a predictor of a worse seizure outcome one year after surgery in patients with temporal lobe epilepsy and mesial temporal sclerosis. Seizure 2012;21:619-623.

38. Metternich B, Wagner K, Brandt A, et al. Preoperative depressive symptoms predict postoperative seizure outcome in temporal and frontal lobe epilepsy. Epilepsy Behav. 2009;16:622-628.

39. Wiebe S, Rose K, Derry P, et al. Outcome assessment in epilepsy: comparative responsiveness of quality of life and psychosocial instruments. Epilepsia 1997;38:430-438. 